\title{
RELATO DE EXPERIÊNCIA DA FORMAÇÃO DE UM GRUPO DE PROMOÇÃO DE SAÚdE NA ESTRATÉGIA DE SAÚDE DA FAMÍLIA
}

\author{
GABRIELA FERNANDA SCHIOCHET ${ }^{1}$, VERIDIANE GEMELLI CHRIST ${ }^{2}$, \\ NAIARA LAGO SOARES ${ }^{3}$, TAYNARA DE SOUZA CARNEIRO ${ }^{4}$, VERA \\ LUCIA LEAL WOSGERAU ${ }^{5}$, MANOELITO FERREIRA SILVA JUNIOR ${ }^{6}$.
}

\begin{abstract}
1 Graduanda em Odontologia pela de Odontologia, Universidade Estadual de Ponta Grossa. gschiochet@hotmail.com.

2 Graduanda em Odontologia pela de Odontologia, Universidade Estadual de Ponta Grossa. veridiane.christ@hotmail.com.

3 Cirurgiã-Dentista formada pela Universidade Estadual de Ponta Grossa. naiara.2013.soares@gmail.com.

4 Mestranda em Odontologia, Universidade Estadual de Ponta Grossa. sctaynara@ hotmail.com.

5 Cirurgiã-dentista da Fundação Municipal de Saúde da Prefeitura Municipal de Ponta Grossa. wosgerauvera@gmail.com.

6 Mestre e Doutor em Odontologia em Saúde Coletiva pela FOP-Unicamp. Pós-doutorando no Programa de Pós-graduação em Ciências da Saúde pela Universidade Estadual de Ponta Grossa (UEPG). Professor Colaborador do Departamento de Odontologia da Universidade Estadual de Ponta Grossa. manoelito_fsjunior@hotmail.com.
\end{abstract}

\section{RESUMO}

A Estratégia de Saúde da Família tem como principal foco as ações de prevenção e promoção à saúde. A formação de redes de apoio tem sido uma estratégia efetiva na interação entre os membros da comunidade para solucionar problemas de natureza individual e coletiva, considerando a necessidade e realidade local. Objetiva-se relatar a formação e a efetivação de grupo de apoio para promoção da saúde na Estratégia de Saúde da Família. O estágio obrigatório extracurricular do curso de Odontologia da Universidade Estadual de Ponta Grossa tem como um dos seus objetivos a elaboração de projeto de intervenção conforme a necessidade local e a governabilidade da equipe de saúde e dos acadêmicos envolvidos. Na Unidade de Saúde da Família Aurélio Grott, Ponta Grossa-PR, foi criado em setembro de 2018, um grupo de tabagismo conforme metodologia preconizada pelo Ministério da Saúde e executado com apoio do Núcleo Ampliado de Saúde da Família. Após o encerramento das cinco cartilhas semanais, houve a realização pelos acadêmicos de avaliação do grupo. Os participantes avaliaram como importante a manutenção do grupo, com atividades desenvolvidas com familiares e de forma quinzenal; no entanto, com outro sem enfoque na cessação do uso do tabaco. O nome do grupo foi discutido e ficou estabelecido como “Mentes Saudáveis". O objetivo do grupo é o crescimento e o desenvolvimento saudável dos participantes e da comunidade. Após 18 meses, o grupo mantém-se ativo, mesmo com interrupção da participação dos acadêmicos no primeiro semestre, com grande adesão e ampla variação de faixa etária dos participantes. O grupo realiza palestras, dinâmicas e colabora com atividades dentro e fora da unidade. O grupo de apoio tem realizado ações de promoção 
de saúde e favorecido o desenvolvimento individual e coletivo, promovendo saúde e qualidade de vida para os acadêmicos, profissionais de saúde e comunidade.

Palavras-chave: Grupos de Autoajuda; Promoção da Saúde; Atenção à Saúde; Estratégia Saúde da Família; Serviços de Integração Docente-Assistencial.

\title{
EXPERIENCE REPORT OF THE FORMATION OF A HEALTH PROMOTION
}

\section{GROUP IN THE FAMILY HEALTH STRATEGY}

\begin{abstract}
The Family Health Strategy focuses on prevention and health promotion actions. The formation of support networks has been an effective strategy in the interaction between members of the community to solve problems of an individual and collective nature, considering the need and local reality. To report the formation and effectiveness of a support group for health promotion in the Family Health Strategy. The mandatory extracurricular internship of the Dentistry course at the State University of Ponta Grossa has as one of its objectives the elaboration of an intervention project according to the local need and governance of the health team and the academics involved. At the Aurélio Grott Family Health Unit, Ponta Grossa-PR, a smoking group was created in September 2018 according to the methodology recommended by the Ministry of Health and executed with support from the Extended Family Health Center. After the closing of the five weekly booklets, the group's evaluation academics conducted them. The participants considered the maintenance of the group as important, with activities carried out with relatives and on a fortnightly basis, however, with another one without a focus on the cessation of tobacco use. The group's name was discussed and was established as "Healthy Minds". The group's objective is the healthy growth and development of the participants and the community. After 18 months, the group remains active, even with the interruption of the students 'participation in the first semester, with great adherence and wide variation of the participants' age range. The group conducts lectures, dynamics and collaborates with activities inside and outside the unit. The support group carried out health promotion actions, and favored individual and collective development, promoting health and quality of life for academics, health professionals and the community.
\end{abstract}

Keywords: Self-Help Groups; Health Promotion; Health Care (Public Health); Family Health Strategy; Teaching Care Integration Services.

\section{INTRODUÇÃO}

O conceito de saúde é amplo, subjetivo e individual, sendo influenciado por fatores como o contexto histórico, político e cultural. No Brasil, a Constituição Federal de 1988 reconheceu a saúde como direito fundamental aos cidadãos e um dever do 
Estado (BRASIL, 1988), determinada pelos Determinantes Sociais, deve ser intermediada por políticas públicas sociais. Em 1990, a implantação do Sistema Único de Saúde (SUS) deu início à organização de um sistema de saúde público e universal, com princípios e diretrizes que abrange todo o território nacional (BRASIL, 1990a; 1990b).

Sendo assim, o cuidado em saúde no Brasil precisava sair do paradigma do modelo biomédico até então vigente e não resolutivo, ou seja, com assistência centrada na doença, no profissional médico, em ambiente hospitalar, com valorização do diagnóstico com uso de exames complementares, e prática terapêutica dependente do uso irrestrito e contínuo de fármacos, para um conceito de atenção à saúde que considerasse o contexto social e a integralidade das ações, no modelo biopsicossocial (DE MARCO, 2006). Nessa fase, a saúde se torna resultado de um processo de produção social que expressa a qualidade de vida de uma população, através da promoção da saúde. Este novo paradigma permite a ruptura dos limites do setor saúde, incorporando, desta forma, a necessidade de uma prática interdisciplinar entre os profissionais e intersetorial (LIMA, 2005).

A Atenção Primária à Saúde (APS), porta de entrada principal dos indivíduos e de suas famílias para o sistema de saúde, conhecida no Brasil como Atenção Básica, passou a conceber, em meados de 1994, a Saúde da Família como o modo prioritário para (re)organização do sistema. Atualmente, a Estratégia Saúde da Família (ESF) trabalha a promoção da saúde como possibilidade de articulação transversal, o que confere visibilidade aos fatores que colocam a saúde da população em risco e às diferenças entre necessidades, territórios e culturas presentes no país, visando à criação de mecanismos que reduzam a vulnerabilidade, defendam a equidade e incorporem a participação e o controle social na gestão das políticas públicas (MOTTA; BASTISTA, 2015).

Os pressupostos para atender as novas demandas na forma de fazer a APS, demonstrou a dicotomia entre a formação profissional e as necessidades de saúde da população brasileira (FAÉ et al, 2016). Sendo assim, a formação de recursos humanos em saúde, também é um dos objetivos do SUS (Brasil, 1990), para melhorar as práticas de saúde, principalmente na valorização da APS e nas ações de promoção de saúde 
(CHIESA et al., 2007). Para isso, a necessidade de parceria entre universidades e serviços de saúde para garantir esses avanços (FAÉ et al, 2016).

A integração ensino-serviço-comunidade é um importante alicerce na construção de um novo modo de ensinar, aprender e fazer, sendo uma atividade efetiva que beneficia todos os sujeitos envolvidos neste processo, ou seja, docentes, discentes, gestores dos serviços de saúde e da educação, profissionais e população (FERRREIRA, 2012). As Diretrizes Curriculares Nacionais (DCN) (BRASIL, 2002) incluem a construção cidadã dos acadêmicos de Odontologia como parte da formação profissional, ou seja, contextualizada e comprometida com a realidade social e epidemiológica através do conhecimento sobre as políticas sociais e de saúde (PELISSARI et al., 2004).

As ações de saúde na APS, e principalmente na ESF, não devem estar focadas apenas no atendimento clínico, mas devem dar ênfase ao planejamento e execução de ações em abordagem coletiva, favorecer o aconselhamento, apoio emocional, apoio educativo e priorizando estratégias grupais e focais para práticas preventivas e voltadas à promoção da saúde. As equipes de saúde ao sair dos serviços de saúde, e promoverem atividades no âmbito da cultura, lazer e educação em espaços comunitários, ou seja, no território vivo, estão promovendo a clínica ampliada. Sendo assim, possibilitam autonomia, resgate de autoestima, ampliação de redes sociais, dentre outros, isto é, uma ação cujo foco não é o sofrimento ou a patologia, mas sim o que é saudável no indivíduo (MARTNS; AMATUZZI, 2012; YOSHIMI et al., 2009). Por isso, o objetivo do presente artigo foi relatar a formação e efetivação de grupo de apoio para promoção da saúde na Estratégia de Saúde da Família.

\section{RELATO DA EXPERIÊNCIA}

A disciplina de estágio supervisionado obrigatório extramuros, Saúde Coletiva II, integralizada no segundo ano do curso de Odontologia da Universidade Estadual de Ponta Grossa (UEPG). Desde o ano de 2017, a disciplina tem desenvolvido projetos de intervenção com o objetivo de fortalecer os vínculos entre ensino-serviço. Os projetos de intervenção foram elaborados de forma conjunta entre os acadêmicos, docentes e equipes de saúde, considerando a realidade local do estágio e a governabilidade e logística de implantação (LUZ et al., 2018). 
Na Unidade de Saúde da Família (USF) Aurélio Grott, no bairro de Los Angeles na cidade de Ponta Grossa, concomitante ao início do segundo semestre de 2018, havia o início do Grupo de Tabagismo que tinha como enfoque o abandono do uso do tabaco. Após convites junto a população, com encontros semanais, durou 5 semanas, segundo o protocolo do Ministério da Saúde, utilizando as cartilhas disponibilizadas em parceria com o Instituto Nacional de Câncer José Alencar Gomes da Silva (INCA), órgão do Ministério da Saúde responsável pelo Programa Nacional de Controle do Tabagismo (PNCT) e pela articulação da rede de tratamento do tabagismo no SUS, em parceria com estados e Distrito Federal e os municípios (INCA, 2020). O projeto foi executado na USF sob a coordenação da Assistente Social, e apoio profissional de uma nutricionista e um Profissional de Educação Física do Núcleo Ampliado de Saúde da Família (NASF), em trabalho conjunto com a Equipe local, através das Agentes Comunitários de Saúde (ACS), principalmente para o convite e apoio durante todos os encontros, da médica para consultar os participantes e passar receita, da enfermeira na avaliação da saúde no primeiro encontro, dos profissionais, da equipe de saúde bucal e dos seus estagiários acadêmicos em Odontologia. Os acadêmicos participaram de todos os encontros como ouvintes e no quarto encontro que tratava dos "Benefícios obtidos após parar de fumar" a palestra ficou a cargo dos acadêmicos que passaram informações sobre os malefícios do consumo do tabaco, mostrando lesões e doenças relacionadas, orientações e formas de escovação dental, utilizando macro modelos, escovas dentais, multimídia incentivando os cuidados da saúde bucal como coadjuvante da qualidade de vida (Figura 1).

Figura 1. Palestra realizada no grupo de tabagismo pelos acadêmicos de Odontologia sobre "Benefícios obtidos após parar de fumar". Ponta Grossa, PR, Brasil, 2018. 


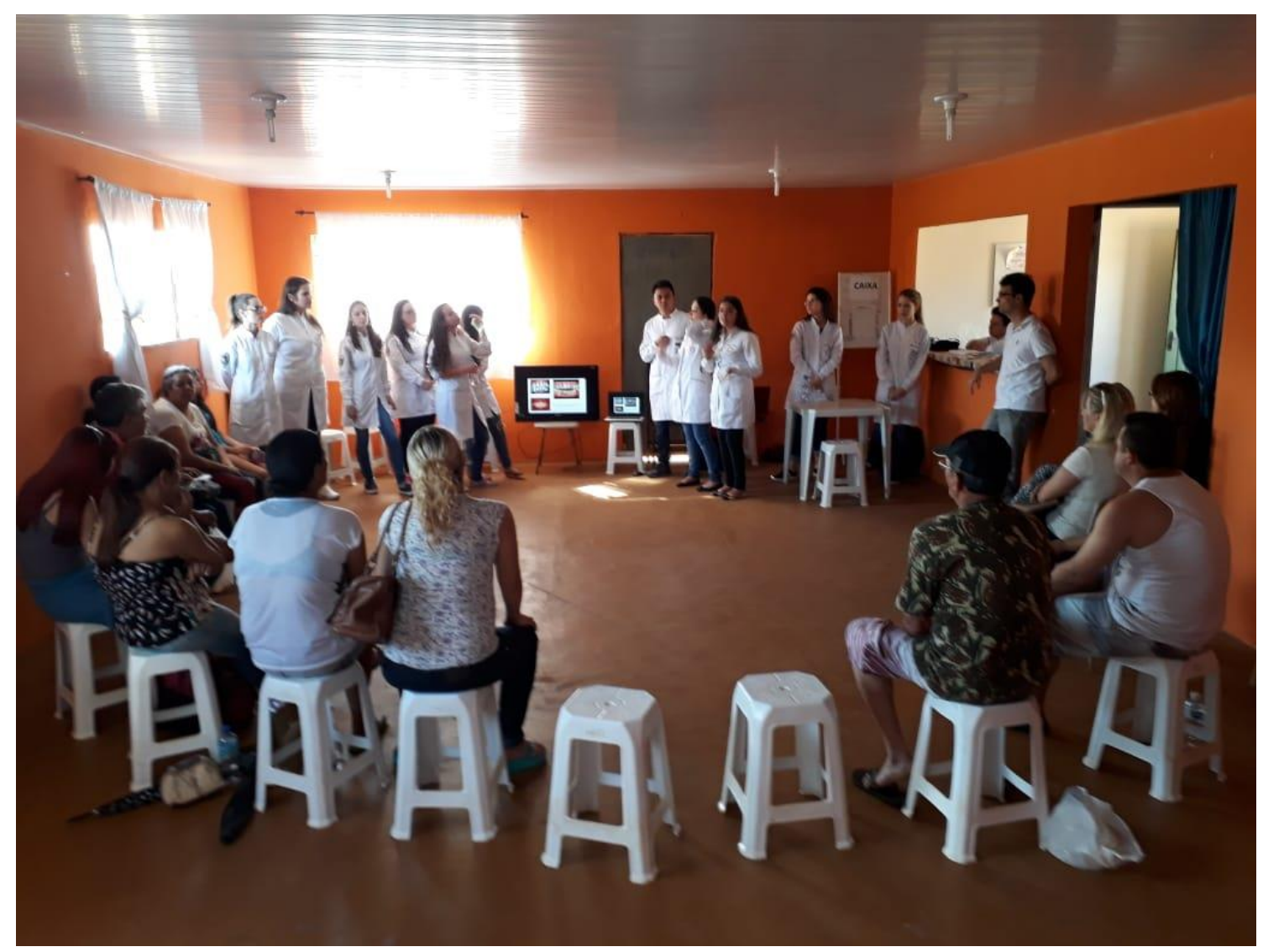

Após o encerramento das cartilhas semanais, no sexto e último encontro, como parte do projeto de intervenção, houve a realização pelos acadêmicos de avaliação da atividade com um questionário qualitativo. Essa avaliação continha questões sobre a avaliação das atividades realizadas e sobre a percepção de necessidade de manutenção do grupo, e qual seria um enfoque mais adequado para o grupo.

Com isso, após discussão no grupo e através da avaliação, o grupo de apoio passou a ter uma ênfase em promoção de saúde, auxiliando-os no desenvolvimento individual e coletivo, promovendo qualidade de vida. O nome do grupo foi discutido e recebeu o nome de “Mentes Saudáveis” e o novo foco ficou voltado ao crescimento e desenvolvimento saudável de cada participante.

Para a realização do projeto, determinou-se um local específico para a abordagem dos temas relacionados ao desenvolvimento saudável, sendo à associação de moradores Jardim Los Angeles decidida como lugar adequado para a realização das atividades. A associação situa-se a uma quadra da Unidade Básica de Saúde Aurélio 
Grott, e é um local especifico para fins educacionais, onde ocorre atividades para comunidade como escolinha de futebol, ballet, capoeira, zumba, aulas de dança gaúcha e violão, e também para reuniões com foco à tentativa de eliminar os problemas socioeconômicos da população adscrita.

Para a realização das atividades todos do bairro são convidados, os encontros são realizadas na associação de moradores Jardim Los Angeles, de quinze em quinze dias, nas segundas-feiras a partir das $13 \mathrm{~h} 30$. Várias atividades foram desenvolvidas ao longo desses 18 meses do grupo (Quadro 1).

Quadro 1. Atividades realizadas durante as reuniões do grupo de apoio "Mentes Saudáveis“. Ponta Grossa, PR, Brasil, 2018-2020.

\begin{tabular}{|c|c|}
\hline Tema & Atividade realizada \\
\hline Projeto 21 dias & $\begin{array}{l}\text { Nesse projeto baseado nas dicas do autor americano Bob Proctor, } \\
\text { ele apresenta a ideia em que se você para com um hábito em um } \\
\text { tempo de } 21 \text { dias, seu corpo e sua mente se acostumam com essa } \\
\text { nova rotina, e você consegue largar determinado hábito (EQUIPE } \\
\text { IBC, 2018). } \\
\text { Para realizar essa atividade, cada pessoa escolhe o hábito que } \\
\text { deseja abandonar, sendo um de cada vez em um período de } 21 \\
\text { dias, e quando realizado ele pode iniciar outro hábito, e se ele } \\
\text { interrompe o hábito e não atinge os } 21 \text { dias, deve se reiniciar o } \\
\text { projeto, desde o dia } 1 \text {. }\end{array}$ \\
\hline $\begin{array}{l}\text { Dinâmica passado } \\
\text { e futuro }\end{array}$ & $\begin{array}{l}\text { Inicialmente foi solicitado para cada participante levar três } \\
\text { objetivos para a reunião. Todos os participantes deveriam deixar } \\
\text { os seus objetos no centro da roda de conversa (Figura 2). A } \\
\text { dinâmica era dividida em dois momentos. Inicialmente cada } \\
\text { indivíduo deveria escolher um objeto (entre todos os objetivos } \\
\text { presentes) que remetesse a alguma lembrança do passado. Após } \\
\text { isso, precisariam justificar os motivos para escolha do objetivo. } \\
\text { No segundo momento, os participantes deveriam escolher um } \\
\text { objeto que tivesse uma relação com o futuro. } \\
\text { O Objetivo dessa dinâmica com os objetos era como cada } \\
\text { simples objeto pode trazer diferentes lembranças e diferentes } \\
\text { histórias, que cada um não deve desistir dos seus desejos para o } \\
\text { futuro e como o que temos atualmente já foi algo que sonhamos } \\
\text { no passado. }\end{array}$ \\
\hline Artesanato & $\begin{array}{l}\text { Em comemoração ao dia das crianças, esse encontro teria como } \\
\text { objetivo fazer com que os mais velhos apresentassem } \\
\text { brincadeiras e brinquedos da sua infância para as crianças. Para a } \\
\text { realização do artesanato desses brinquedos de antigamente foi } \\
\text { pedido para que os participantes do grupo levassem garrafas pet, }\end{array}$ \\
\hline
\end{tabular}




\begin{tabular}{|c|c|}
\hline & $\begin{array}{l}\text { tesoura, tampas de garrafa, barbante, cola, jornal, fita, latas, tinta } \\
\text { guache, palitos de churrasco, etc. O resultado do artesanato foi a } \\
\text { discussão das brincadeiras antigas com as atuais e o conflito de } \\
\text { gerações em relação as brincadeiras e brinquedos antigos e a } \\
\text { tecnologia atual, além do desenvolvimento da criatividade e da } \\
\text { atividade em grupo (Figura } 3 \text { ). }\end{array}$ \\
\hline $\begin{array}{l}\text { Campanhas de } \\
\text { Saúde }\end{array}$ & $\begin{array}{l}\text { O grupo de apoio realiza atividades específicas nas Campanhas } \\
\text { desenvolvidas pela equipe, por exemplo, Outubro Rosa, } \\
\text { Novembro Azul. O intuito é incluir os participantes nas } \\
\text { campanhas, favorecer as idas contínuas ao serviço de saúde, e } \\
\text { além disso, favorecer uma reflexão sobre as temáticas e } \\
\text { campanhas que estão sendo desenvolvidas pela Equipe de Saúde. } \\
\text { E inclusive, a inclusão dos participantes como multiplicadores de } \\
\text { informação sobre as atividades realizadas para a comunidade. }\end{array}$ \\
\hline $\begin{array}{l}\text { Práticas } \\
\text { Integrativas e } \\
\text { Complementares }\end{array}$ & $\begin{array}{l}\text { O grupo convida terapeutas para realização de Práticas } \\
\text { Integrativas e Complementares (PIC), com profissionais do } \\
\text { serviço público ou não. O município ainda não apresenta uma } \\
\text { política específica, mas está em andamento. } \\
\text { As terapias se baseiam na visão holística, ou seja, que trata } \\
\text { problemas e doenças a partir de uma visão global e faz uma } \\
\text { análise da pessoa como um todo. O objetivo é ajudar o paciente a } \\
\text { melhorar a qualidade de vida e chegar ao seu ponto máximo de } \\
\text { equilíbrio físico, emocional e energético (SIGNIFICADOS, } \\
\text { 2019). Os resultados dessas terapias são maior conhecimento } \\
\text { pessoal, cura espiritual, relaxamento e maior desenvolvimento } \\
\text { mental e saudável dos participantes (Figura 4). }\end{array}$ \\
\hline $\begin{array}{l}\text { Dinâmicas em } \\
\text { grupo }\end{array}$ & $\begin{array}{l}\text { A maioria das atividades realizadas são nessa modalidade. Com o } \\
\text { objetivo de aplicar a vivência do tema proposto, através das } \\
\text { dinâmicas e realizar atividades em grupo, ampliando o } \\
\text { conhecimento e a relação entre os participantes, momentos } \\
\text { descontraídos para uso de outros recursos ou dinâmicas, tais } \\
\text { como: atividade com balão, cadeiras, "atravessar o rio" e } \\
\text { dinâmica das mãos dadas, e com isso demonstrar a importância } \\
\text { dessa união tanto nas dinâmicas realizadas como no dia a dia de } \\
\text { cada participante. Buscando aplicar de forma sutil o método da } \\
\text { resolução de problemas baseado no Arco de Maguerez (MELO et } \\
\text { al., 2015). } \\
\text { Todas as semanas também são utilizados curingas para as } \\
\text { dinâmicas em grupo sobre autor estima (ALMEIDA, SOUZA, } \\
\text { 2001). }\end{array}$ \\
\hline
\end{tabular}


Figura 2. Objetos trazidos pelos participantes para a realização da dinâmica "Passado e futuro". Ponta Grossa, PR, Brasil, 2019.

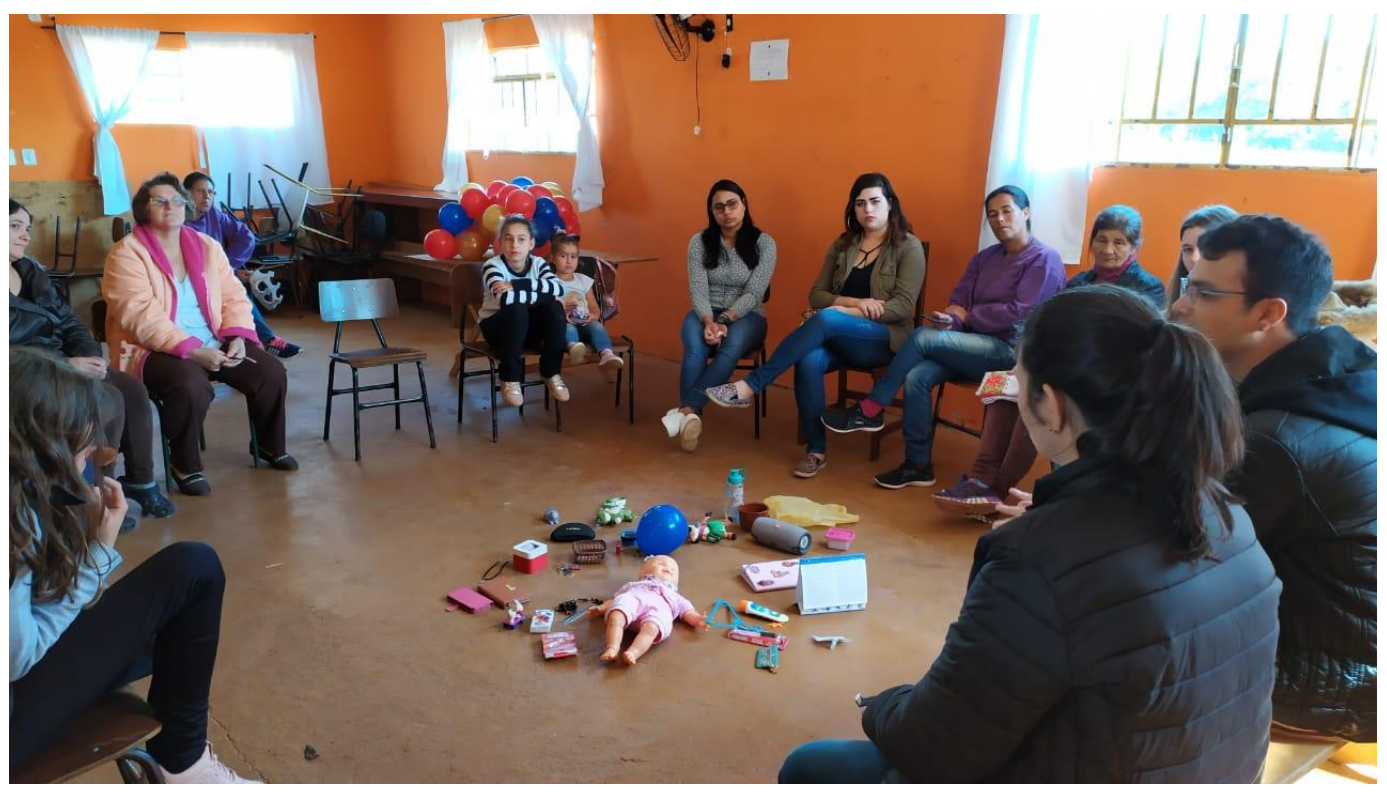

Figura 3. Artesanato realizado no encontro em comemoração ao dia das crianças. Ponta Grossa, PR, Brasil, 2019.

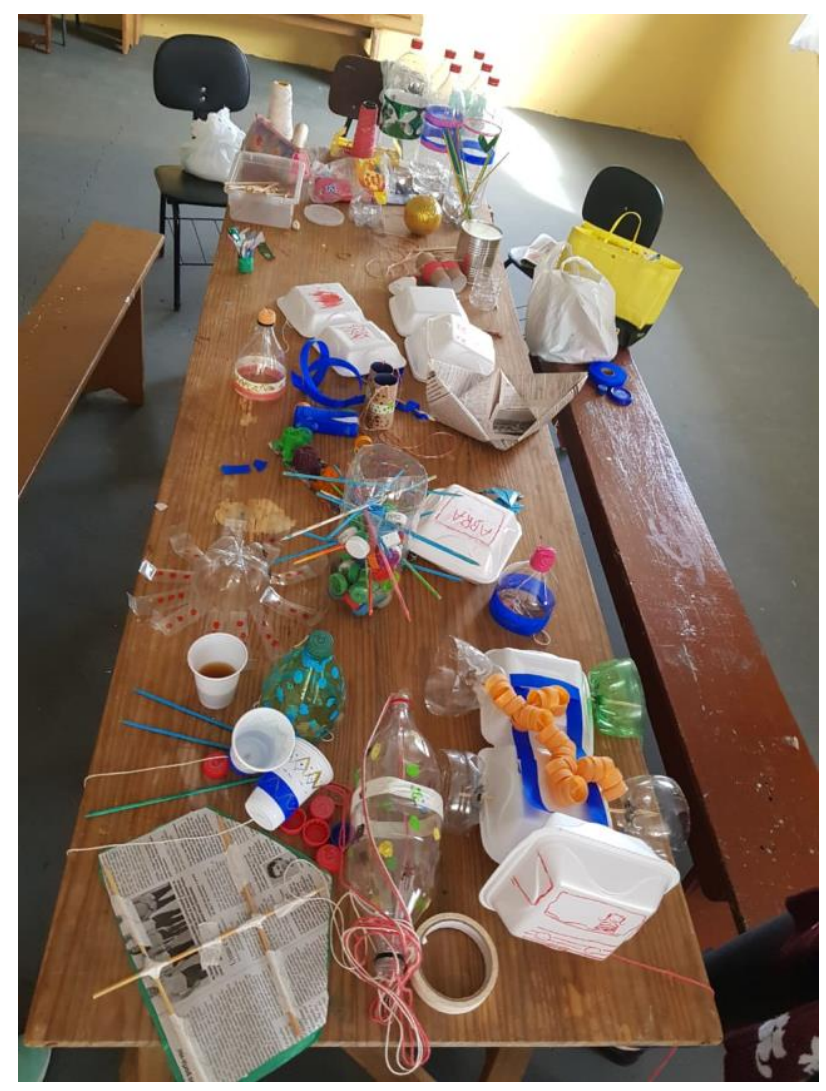


Figura 4. Prática de terapia em grupo. Ponta Grossa, PR, Brasil, 2018.

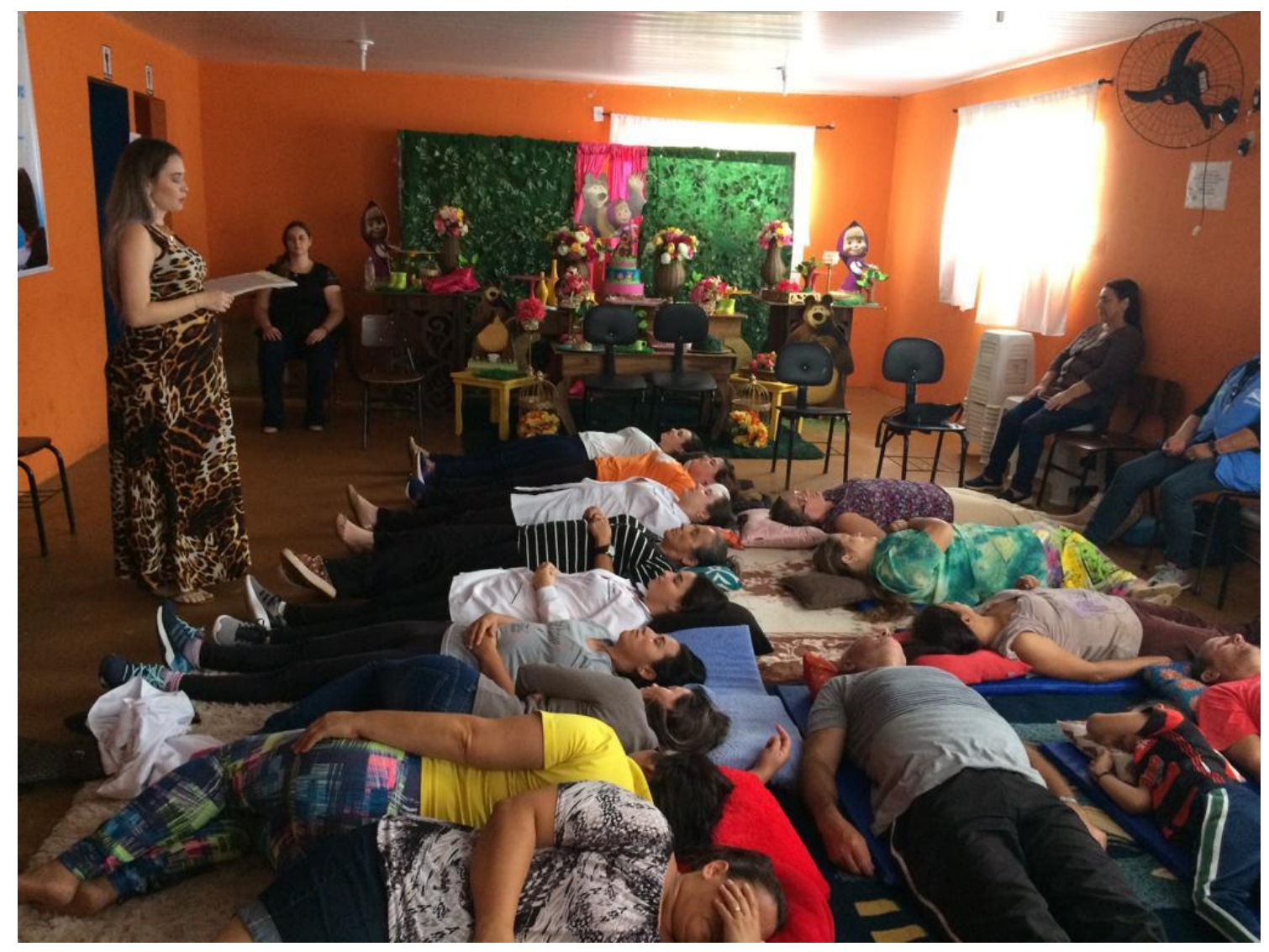

Para auxiliar na participação de novos membros no grupo, são confeccionados convites individuais que são distribuídos para população local, na própria Unidade de Saúde Aurélio Grot e na associação de moradores, ou pelas Agentes Comunitária de Saúde (ACS) durante as visitas domiciliares. Além disso, um cartaz sempre é confeccionado e colado no mural da USF e na associação de moradores.

Visando a manutenção da adesão dos participantes do grupo, foi proposto a criação de um grupo de WhatsApp, o qual é utilizado para envio de vídeos motivacionais, compartilhamento de informações de saúde, recados sobre as reuniões ou lembrança de alguma atividade, além do envio de frases motivacionais diárias para que os participantes consigam atingir a meta do projeto 21 dias.

Após 12 meses, o grupo manteve-se ativo, mesmo com interrupção da participação dos acadêmicos no primeiro semestre de 2019. Com grande adesão e ampla 
variação de faixa etária dos participantes, o grupo realiza palestras, dinâmicas, tira dúvidas e colabora com atividades da unidade.

A atuação desse grupo é uma estratégia de desenvolvimento tanto individual, quanto coletivo, promovendo saúde e qualidade de vida dos membros da comunidade, como também dos acadêmicos de Odontologia e equipe de saúde participantes.

\section{DISCUSSÃO}

O grupo "Mentes saudáveis" tem sido uma experiência exitosa de articulação ensino-serviço-comunidade na efetivação de atividades que promovem saúde de forma contínua, vinculado ao serviço de atenção básica em uma perspectiva do território vivo e na clínica ampliada. Através de oficinas de interação entre os participantes, tais como: dinâmicas de grupo, brincadeiras, artesanatos, culinária, são discussão de problemáticas individuais e coletivas advindas da população, e o grupo de apoio busca incentivar e realizar melhores condições de vida da população, através do crescimento e desenvolvimento de cada participante e da comunidade.

A articulação conjunta entre Universidade e Serviços de Saúde, principalmente utilizando de projeto de intervenção como parte dos estágios dos acadêmicos de Odontologia, tem incentivado e estimulado a formação de grupos de apoio, e tornou-se algo inovador para remodelar e melhorar o cuidado em saúde oferecido na APS do município. Apesar dos grupos de tabagismo não ser uma nova atividade desenvolvida na APS, a inclusão de uma etapa de avaliação do serviço prestado prevista no projeto de intervenção na presente USF, mudou o modo de agir da equipe de saúde e no planejamento do projeto futuro. O envolvimento dos profissionais em conjunto com a comunidade determinou a verificação da necessidade de formação de grupo contínuo, e com novo enfoque. Sendo assim, apesar do NASF findar o seu trabalho de apoio durante o grupo de tabagismo, a USF permaneceu com o trabalho junto o grupo, no intuito de vínculo longitudinal e efetivação de um grupo que respondesse outras demandas priorizadas pela população local.

Vale destacar ainda aqui, o trabalho em equipe interdisciplinar na implantação do grupo de tabagismo, e da articulação existente no município entre a Atenção Básica e 
os NASF, em prol do objetivo comum de favorecer a saúde da população. Assim, temse verificado que as medidas legislativas, econômicas e educativas desenvolvidas no Brasil na última década, tem sido efetiva na redução no uso do tabaco (INCA, 2020).

No presente grupo, houve participação conjunta da comunidade nas decisões de interesse para os indivíduos do próprio grupo, por exemplo, sobre a periodicidade quinzenal de atividades, e assim, cada uma das diversas atividades são previamente escolhidas pelos participantes, e podem ser previamente planejadas pelos profissionais responsáveis. As atividades além de ser um elo de interação entre a comunidade, tem um foco na educação em saúde como ferramenta de promoção de saúde, pois proporciona aos indivíduos o resgate de sua autonomia, a percepção de valores e conhecimentos, o desenvolvimento de uma visão crítica e do empoderamento da população (SANTOS et al., 2012). Além disso, o presente grupo, oferece atividades com diferentes metodologias, principalmente pela diversidade dos seus participantes, ou seja, o grupo não enfoca em ter um público-alvo estabelecido e com idade prédeterminada, tornando assim mais atrativo a participação no grupo para todos.

Através da implantação, efetivação e manutenção desse grupo de apoio, têm-se verificado um fortalecimento na relação entre comunidade e os profissionais de saúde atuantes na Unidade Básica de Saúde. A aproximação, envolvimento e o interesse, tem construído uma relação interpessoal de confiança para ambas as partes e pode ser fruto da valorização na participação comunitária e no protagonismo que os indivíduos tem recebido (CARDOSO et al., 2013). A participação da comunidade no trabalho da ESF por meio dos grupos locais organizados converge para afirmar a implementação de uma nova proposta de atenção a saúde. Proposta que almeja intervir em situações de risco para amenizar os efeitos deletérios do processo de desenvolvimento dos povos (CARDOSO et al., 2013). Como o grupo relatado, onde através de uma decisão dos membros da comunidade participantes, junto com os profissionais envolvidos e os estagiários, decidiram manter o grupo e mudar o foco para um melhor desenvolvimento e crescimento saudável dos indivíduos envolvidos, inclusive dos próprios profissionais de saúde, como dos acadêmicos estagiários.

Como uma atividade inovadora, ressalta-se ainda a relação entre professores, alunos, gestores, profissionais de saúde, e comunidade. O estágio no SUS é ainda recente, e está em permanentemente evolução (BULGARELI et al., 2014; FAÉ et al., 
2016), mas ressalta no papel determinante em atingir as Diretrizes Curriculares Nacionais (DCN) do Curso de Graduação em Odontologia (BRASIL, 2002), pois espera a formação de um profissional crítico, reflexivo e capaz de responder as necessidades reais da população brasileira. E para isso, devem atuar multiprofissionalmente, interdisciplinarmente e transdisciplinarmente com extrema produtividade na promoção da saúde baseado na convicção científica, de cidadania e de ética. É inerente os benefícios para o acadêmico, como o SUS tem-se configurado como meio apropriado para o aprendizado e impactado na formação de profissionais da saúde além de boas habilidades técnicas e clínicas, mas também humanos e sensíveis à saúde bucal brasileira e com mais autonomia, preparo e seguro para o enfrentamento das dificuldades da profissão e do mercado de trabalho (BULGARELI et al., 2014).

O estágio em Odontologia na atenção básica apresenta múltiplas possibilidades ao acadêmico (SILVA-JUNIOR et al., 2015). No entanto, ressaltar aqui, que o objetivo do estágio supervisionado realizado pela Universidade Estadual de Ponta Grossa, no segundo ano da graduação em Odontologia, não tem enfoque clínico, mas objetiva apresentar o território, o funcionamento e o processo de trabalho de uma equipe de saúde, principalmente na Estratégia de Saúde da Família. A importância de uma vivência com trabalhadores do SUS, aprendendo a lógica do trabalho em equipe multiprofissional, favorece aos acadêmicos espaços para discussões e desenvolvimento de boas práticas e relações no cuidado aos usuários (BULGARELI et al., 2014). Neste estágio, além do papel de observador, incluiu-se uma etapa de atuação ao acadêmico, através do planejamento e execução do projeto de intervenção (LUZ et al., 2018). E por isso, o estágio na UEPG visa estabelecer meios para conhecer a realidade e interferir de alguma forma para aprimorar o sistema. Esse formato de estágio, tem se mostrado efetivo, e por isso, está sendo incentivado por meio desse relato, pode ser facilmente incorporado em outros cursos e municípios brasileiros.

\section{CONSIDERAÇÕES FINAIS}

O presente relato, inicialmente com a formação de um grupo de tabagismo, que seguiu as recomendações do Ministério da Saúde, em uma atividade articulada entre a 
Unidade de Saúde da Família, o Núcleo Ampliado de Saúde da Família e estagiários do curso de Odontologia, após finalização e avaliação, foi mantido, no entanto, com novo enfoque, conforme a vontade dos participantes.

As reuniões do grupo de promoção de saúde, com responsabilidade da Equipe de Saúde da Família e dos estagiários de Odontologia, apresentam encontros quinzenais, sem faixa etária definida, com dinâmicas e atividades diversas, previamente escolhidas pelos participantes. Após o período de 18 meses de formação, o grupo de apoio tem favorecido e efetivado o desenvolvimento individual e coletivo, promovendo saúde e qualidade de vida para todos os atores envolvidos, acadêmicos, profissionais de saúde e comunidade.

\section{REFERÊNCIAS}

ALMEIDA N.; SOUZA, M. Curingas para minha auto-estima: uma nova consciência nas cidades. Curitiba: Gráfica Radial; 2001. 174 p.

BRASIL. Constituição (1988). Constituição da República Federativa do Brasil. Brasília, DF: Senado Federal; 1988.

BRASIL. Lei $n^{\circ}$ 8.080, de 19 de Setembro de 1990. Dispõe sobre as condições para a promoção, proteção e recuperação da saúde, a organização e o funcionamento dos serviços correspondentes e dá outras providências. Diário Oficial da União, 20 Set 1990.

BRASIL. Resolução Conselho Nacional de Educação/Câmara de Educação Superior $\mathrm{n}^{\circ}$ 3, de 19 de fev. 2002. Institui as Diretrizes Curriculares Nacionais do Curso de Graduação em Odontologia. Diário Oficial da União, 04 Mar 2002.

BULGARELLI, A. F.; SOUZA, K. R.; BAUMGARTEN, A.; SOUZA, J. M.; ROSING, C. K.; TOASSI, R. F. C.; Formação em saúde com vivência no Sistema Único de Saúde (SUS): percepções de estudantes do curso de Odontologia da Universidade Federal do Rio Grande do Sul (UFRGS), Brasil. Interface: Comumicação, Saúde e Educação, v. 18, n. 49, p. 351-62, 2014.

CARDOSO, L. S.; CEZAR-VAZ, M. R.; COSTA, V. Z.; BONOW, C. A.; ALMEIDA, M. C. V. Promoção da saúde e participação comunitária em grupos locais organizados. Revista Brasileira de Enfermagem (REBEn), v. 66, n. 6, p. 928-934, 2013. Disponível em: 〈http://www.scielo.br/pdf/reben/v66n6/18.pdf>. Acesso em: 21 mar. 2020. 
CHIESA, A. M.; NASCIMENTO, D. D. G.; BRACCIALLI, L. A. D.; OLIVEIRA, M. A. C.; CIAMPONE, M. H. T. A formação de profissionais da saúde: aprendizagem significativa à luz da promoção da saúde. Revista Cogitare Enfermagem, v.12, n. 2, p. 236-240, 2007.

DE MARCO, M. A. Do modelo biomédico ao modelo biopsicossocial: um projeto de educação permanente. Revista Brasileira de Educação Medica. v.30, n.1, p.60-72, 2006.

FAE, J. M. ; SILVA JUNIOR, M. F. ; CARVALHO, R. B. ; ESPOSTI, C. D. D.; PACHECO, K. T. S. . A integração ensino-serviço em Odontologia no Brasil. Revista da Abeno, v. 16, n. 3, p. 7-18, 2016. Disponível em: $<$ https://revabeno.emnuvens.com.br/revabeno/article/view/286/242>. Acesso em: 19 mar. 2020.

FERREIRA, V. S.; BARRETO, R. L. M.; OLIVEIRA, E. K.; FERREIRA, P. R. F.; SANTOS, L. P. S.; MARQUES, V. E. A. et al. PET-Saúde: uma experiência prática de integração ensino-serviço-comunidade. Revista Brasileira de Educação Médica, v. 36, n.1, p. 147-151, 2012.

IBC. Conheça a teoria dos 21 dias. 2018. Disponível em: $<$ https://www.ibccoaching.com.br/portal/comportamento/conheca-teoria-dos-21-dias/>. Acesso em: 18 mar. 2020.

INCA. Tratamento do tabagismo. 2020. Disponível em: <https://www.inca.gov.br/programa-nacional-de-controle-do-tabagismo/tratamento>. Acesso em: 19 mar. 2020.

LIMA, M. Atuação pscicológica coletiva: uma trajetória profissional em unidade básica de saúde. Psicologia em Estudo, v. 10, n. 3, p. 431-440, 2005. Disponível em: $<$ http://www.scielo.br/scielo.php?pid=S1413-

73722005000300011\&script=sci_abstract\&tlng=pt $>$. Acesso em: 18 mar. 2020.

LUZ, M. C.; BALDANI M. H.; SILVA JUNIOR, M. F. Integração ensino-serviçocomunidade no curso de odontologia: experiências exitosas entre o município e a universidade. Archives of health investigation, v. 7, p. 84, 2018. <Disponível em: <http://www.archhealthinvestigation.com.br/ArcHI/article/view/3826/pdf >. Acesso em: 20 mar. 2020.

MARTINS, K.; AMATUZZI, M. Experiências comunitárias: repensando a clínica psicológica no SUS. Psicologia \& Sociedade, v. 14, n. 3, 2012. Disponível em: < http://www.scielo.br/scielo.php?script=sci_arttext\&pid=S0102-71822012000300020>. Acesso em: 18 mar. 2020.

MELO, M. C.; BOECKMANN, L. M. M.; COSTA, A. R. C.; MOURA, A. S.; GUILHEM, D. Aprendizagem baseada na problematização: utilizando o Arco de Maguerez na graduação de enfermagem. Revista Eletrônica Gestão \& Saúde, v. 7, n. 
1, p. 247-259. 2015. Disponível em: <https://periodicos.unb.br/index.php/rgs/article/view/3410>

MOTTA, L. C. D. S.; BATISTA, R. S. Estratégia Saúde da Família: Clínica e Crítica. Revista Brasileira de Educação Médica, v. 39, n. 2, 2015. Disponível em: <http://www.scielo.br/scielo.php?script=sci_arttext\&pid=S0100-55022015000200196>. Acesso em: 18 mar. 2020.

PELISSARI, L. D.; BASTING, R. T.; FLÓRIO, F. M. Vivência da realidade: o rumo da saúde para a odontologia. Revista da Abeno, v. 5, n. 1, p. 32-9, 2004.

SANTOS, K. T. D.; PACHECO FILHO, A. C.; GARBIN, C. A. S. Educação em saúde bucal na visão de acadêmicos de Odontologia. Arquivos em Odontologia, v. 48, n. 2, p. 96-101, 2012. Disponível em: <http://revodonto.bvsalud.org/scielo.php?pid=S151609392012000200006\&script=sci_a rttext>. Acesso em: 21 mar. 2020.

SIGNIFICADOS. Significado de Terapia Holística. 2019. Disponível em: <https://www.significados.com.br/terapia-holistica/>. Acesso em: 18 mar. 2020.

SILVA-JUNIOR, M. F.; PACHECO, K. T. S.; CARVALHO, R. B. Multiplicidade de atuações do acadêmico de Odontologia no estágio curricular: relato de experiência. Arquivos em Odontologia, v. 51, n. 4, p. 194-204, 2015. Disponível em: $<$ http://revodonto.bvsalud.org/scielo.php?script=sci_arttext\&pid=S151609392015000300004\&lng=en\&nrm=iso>. Acesso em: 20 mar. 2020.

YOSHIMI, O.; RIBEIRO, E. L. Ações de saúde mental na atenção básica: caminho para ampliação da integralidade da atenção. Ciência e Saúde Coletiva, v. 14, n. 2, p. 477486, 2009. Disponível em: http://www.scielo.br/scielo.php?script=sci_arttext\&pid=S141381232009000200016>. Acesso em: 18 mar. 2020. 